\title{
A Routing for Delay-sensitive Traffic in Aerospace Networks
}

\author{
Weiqiang $\mathrm{Wu}^{1,2, a}$, Sunan Wang ${ }^{1, \mathrm{~b}}$ and Rui Zhang ${ }^{1, \mathrm{c}}$ \\ 'Shenzhen Polytechnic, Shenzhen, Guangdong, 518055, China \\ ${ }^{2}$ Harbin Institute of Technology Shenzhen Graduate School, Shenzhen, Guangdong, 518055, \\ China \\ awwq520516@163.com \\ bszpt.wsn@qq.com \\ `zhangrui@szpt.edu.cn
}

\begin{abstract}
Keywords: aerospace network, routing protocol, delay-sensitive, shortest delay path.
Abstract. To guarantee the OoS of delay-sensitive traffic and improve the routing performance of the aerospace network, the shortest delay routing protocol for delay-sensitive traffic is proposed. Base on the queue information and packet loss probability, the routing protocol can provide the shortest delay path for the delay-sensitive traffic. Simulation shown that, compared to the traditional shortest path routing, the shortest delay routing can reduce the end-to-end delay of delay-sensitive traffic and can improve the network performance.
\end{abstract}

\section{Introduction}

Aerospace network is a complex network with large coverage. A variety of satellites and space platforms play the roles of the backbones of the network and forward the data for the users such as aircrafts and the terminals on ground. Because of the large coverage, the performance of data transmission in this multi-hop network is effected by routing protocol.

According to the method on solving problem of the dynamic changing topology, the classic routing for aerospace network can be classified as routing based on virtual topology[1], routing based on virtual region[2,3], on-demand routing[4,5], and routing for multi-layer satellite networks[6,7]. The main purpose of these routings is to avoid the effect on data delivery caused by the movement and switch of satellites and to guarantee the basic data transmission.

Because of the wide range of application and large coverage of the aerospace network, it has to deliver data for variety of users with more diversity. Therefore, to gurantee the QoS, some routing protocols based on remaining bandwidth and link duration are proposed to optimize the data path for some sigle traffic type[8,9]. To optimize the data path of different traffic types, the routing based on traffic types is proposed in [10]. It establishes routing tables for each type of traffic, which needs large storage space. To improve the performance or decuce the complexity, some new routing protocls are proposed recent years[11-13].

In aerospace network, the distance between the backbones such as satellites and space platforms is fairly large which can be thousands $\mathrm{km}$. The long distance causes large delay in each space link. Therefore the end-to-end delay of data transmission is large. For some delay-sensitive traffic such as the voice and control message, routing protocol has to provide a optimal path with the shortest end-to-end delay. So the distance between nodes has been considered as one of the most improtant metrics in many proposed routings. As we know, the end-to-end delay contains propogation delay, queuing delay and the transmission delay. The queue methord and queuing delay of delay-sensitive traffic have to be considered in order to decuce the end-to-end delay. Because of the noise and interference in space, the packet loss can not be ignored. And if the packet is dropped, it has to be retransmitted. Because of the long distance of each link in aerospace network, the delay caused by retransmitted can not be ignored. So when the routing computes the propogation delay, the retransmission delay has to be invoved. 
In this paper, a shortest delay routing protocol for aerospace is proposed. Base on the queue information and packet loss probability, the routing protocol can provide the shortest delay path for the delay-sensitive traffic to guarantee its QoS.

\section{Queuing Delay}

Queuing Model. In this paper, we deal with the queuing delay based on queuing theory. The packets of different traffics which are going to be forwarded queue in each backbone. We divide traffics into two types which are "delay-sensitive traffic" and "delay-insensitive traffic". The end-to-end delay of delay-sensitive traffic such control messages, voice and commands should be reduced as far as possible. The queuing model is shown in Fig. 1.

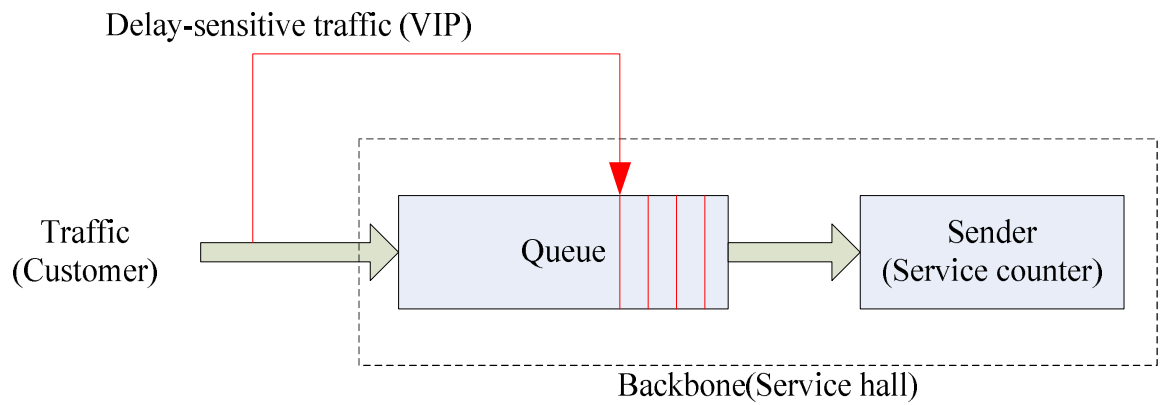

Fig. 1 The queuing model for traffics

In this queue model, delay-sensitive traffic has high priority which is similar to the VIP. The packet of delay-sensitive traffic can jump to the front of the queue. Delay-insensitive traffic has low priority which has to be the tail of the queue.

Queuing Time. The arrival of delay-sensitive traffic follows Poisson distribution with parameter $\lambda_{1}$, and the delay-insensitive traffic follows Poisson distribution with parameter $\lambda_{2}$. All the traffic arrival parameter is $\lambda=\lambda_{1}+\lambda_{2}$. The service time is $X=1 / \mu$ which is also the transmission delay, where $\mu$ is the service rate. According to queuing theory, the average service time is denoted as Eq. 1.

$$
\bar{X}=E(X)=\sum_{i=1}^{2} \frac{\lambda_{i}}{\lambda} X=\frac{\sum_{i=1}^{2} \frac{\lambda_{i}}{\mu}}{\lambda}=\frac{\sum_{i=1}^{2} \rho_{i}}{\lambda}=\frac{1}{\mu}
$$

The average remaining service time is denoted as Eq. 2 .

$$
\overline{S_{e}}=E\left(S_{e}\right)=\frac{E\left(X^{2}\right)}{2 E(X)}=\frac{\sigma^{2}+(\bar{X})^{2}}{2 \bar{X}}=\frac{1}{2 \mu}
$$

Where $\sigma^{2}$ is 0 . The average queuing time for delay-sensitive traffic is denoted as Eq. 3, where $\rho_{i}=\frac{\lambda_{i}}{\mu}$.

$$
W_{q 1}=\rho_{1} W_{q 1}+\rho \overline{S_{e}}=\frac{\rho}{2 \mu\left(1-\rho_{1}\right)}
$$

When the routing the shortest delay path for delay-sensitive traffic, the $W_{q 1}$ should be added into the link delay metric. 


\section{Retransmission Delay}

When $D$ is the propagation delay which is decided by the distance between two backbones, $p$ is the link packet loss rate, a packet can be successfully forwarded to next backbone through $k$ times transmission, and $K$ is random variable that means the transmission times. The probability of $K$ is denoted as Eq. 4.

$$
P\{X=k\}=p^{k-1}(1-p)
$$

The average transmission times by a link is denoted as Eq. 5 .

$$
E(X)=\frac{1}{1-p}
$$

Involved the delay caused by retransmission, the average propagation delay in a link is denoted as Eq. 6.

$$
D_{\text {ave }}=D^{*} \frac{1}{1-p}=\frac{D}{1-p}
$$

\section{Shortest Delay Routing}

The network can be abstracted as a weighted directed graph $G(V, E)$. Where $V$ is the vertex, which means the set of nodes in the network, $V=\left\{V_{l}, V_{2}, \ldots, V_{n}\right\}$. E is the edge of the graph, which means the links between nodes, $E=\left\{\left(V_{l}, V_{2}\right),\left(V_{l}, V_{3}\right), \ldots,\left(V_{i}, V_{j}\right)\right\}, D_{i j}$ is the delay of link $\left(V_{i}, V_{j}\right)$. According to the analysis above, $D_{i j}$ is denoted as Eq. 7.

$$
D_{i j}=W_{q 1_{j}}+D_{a v g_{i j}}+1 / \mu_{j}
$$

When $s$ is the source node and $d$ is the destination node, $p(s, d)$ is a data path from $s$ to $d$, which consists some links. $R$ is the set of the nodes which in $p(s, d) . C(p(s, d))$ is the cost when data is transmitted through the path $p(s, d)$.

For delay-sensitive traffic, the path with the shortest end-to-end delay is optimal. The $C(p(s, d))$ is end-to-end delay denoted as Delayend-to-end, which is the duration time from the data generation in the source to reception in destination. The shortest delay routing is to minimize the $C(p(s, d))$ which is denoted as Eq. 8 by choosing the optimal path.

$$
C(p(s, d))=\sum_{i j \in p(s, d)} D_{i j}=\sum_{i j \in p(s, d)}\left(W_{q 1_{j}}+D_{a v g_{i j}}+1 / \mu_{j}\right)
$$

By updating the $\lambda, \lambda_{1}$ and $\mu$ of each backbone node and the packet loss rate of each link $p$, the backbones can get the whole environment of the network, and choose the best path for delay-sensitive traffic. The process of shortest delay routing is shown in Fig. 2. 


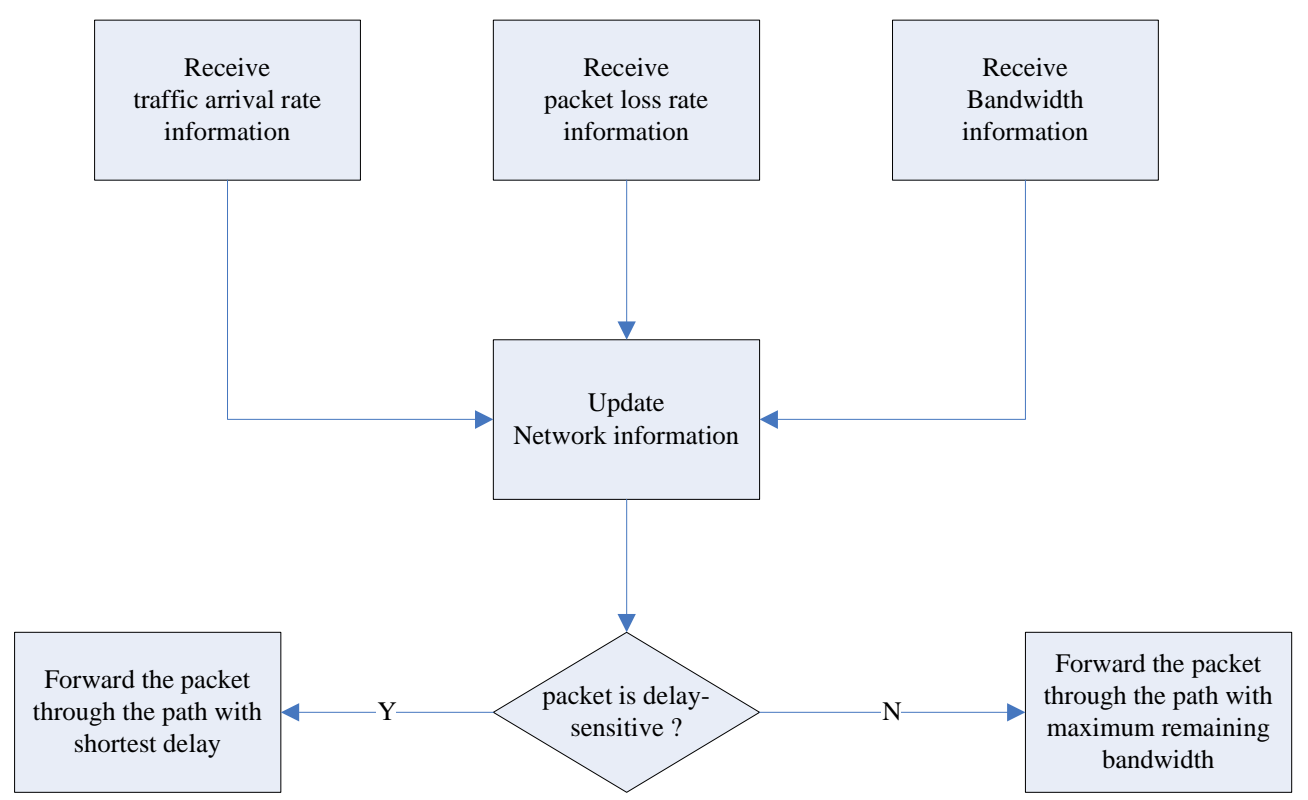

Fig. 2 The process of shortest delay routing

\section{Simulations}

To analyze the performance of the routing, we set Iridium satellite constellation and 14 nearspace platforms whose heights are $20 \mathrm{~km}$ and $500 \mathrm{~km}$ far from its neighbor. 600 traffics is generated randomly in this network which follows Poisson distribution with $\lambda=6$, in which $20 \%$ are delay-sensitive traffic. The packet size is $1 \mathrm{~KB}$, and the bandwidth of each link is $25 \mathrm{Mbps}$. Packet loss rate follows uniform distribution with parameter $[0,0.2]$. The performance for end-to-end delay is shown in Fig. 3, and the performance for network throughput is shown in Fig. 4.

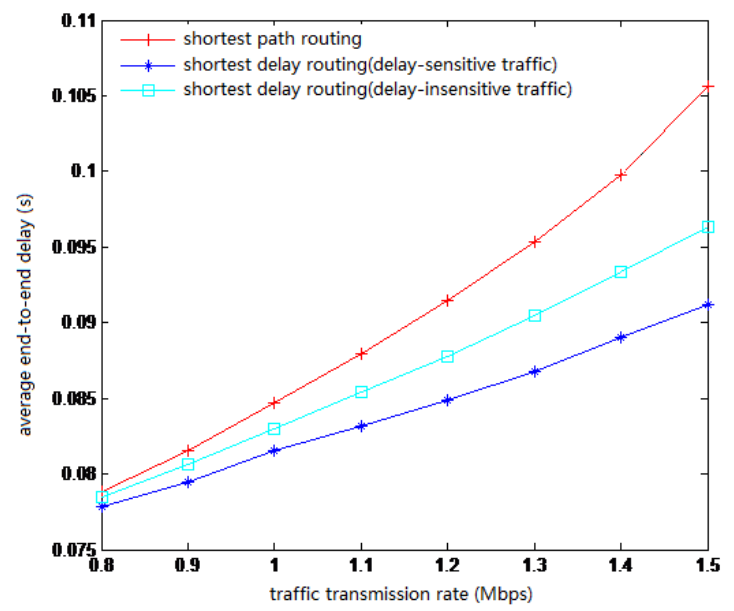

Fig. 3 The average end-to-end delay

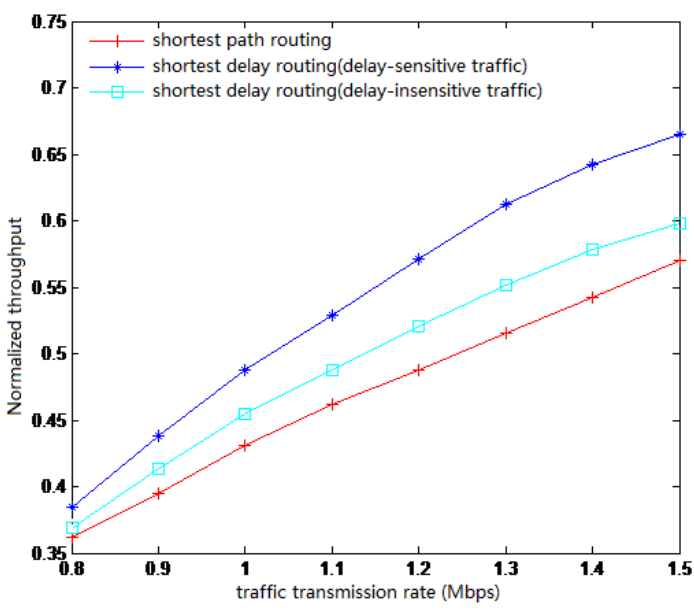

Fig. 4 The network throughput

The end-to-end delay of shortest delay routing is much shorter than that of the traditional shortest path routing. And the end-to-end delay of delay-sensitive traffic is reduced as far as possible. Meanwhile, because of shorter end-to-end delay, the throughput of delay-sensitive is increased. The performance of delay-sensitive traffic is improved by shortest delay routing.

The average hops is shown in Fig. 5 and the load balance index is shown in Fig. 6. Compared to shortest path routing, shortest delay routing can make the load balance better and the average hops of the path is not much more. 


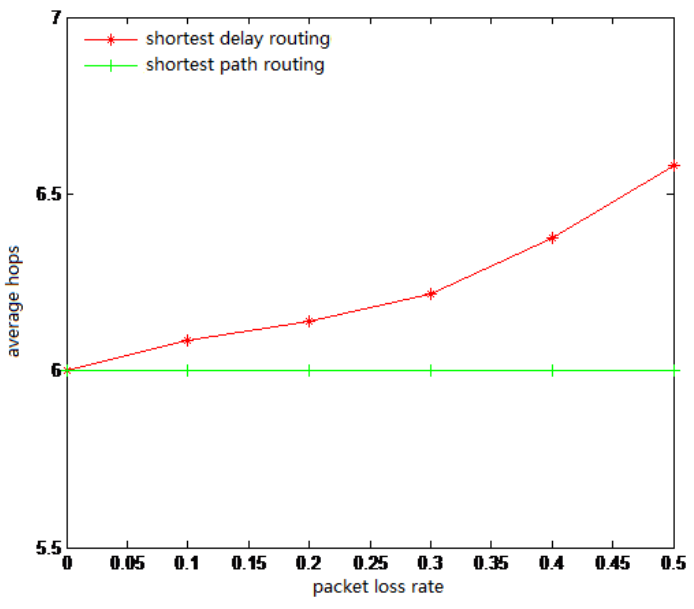

Fig. 5 The average hops

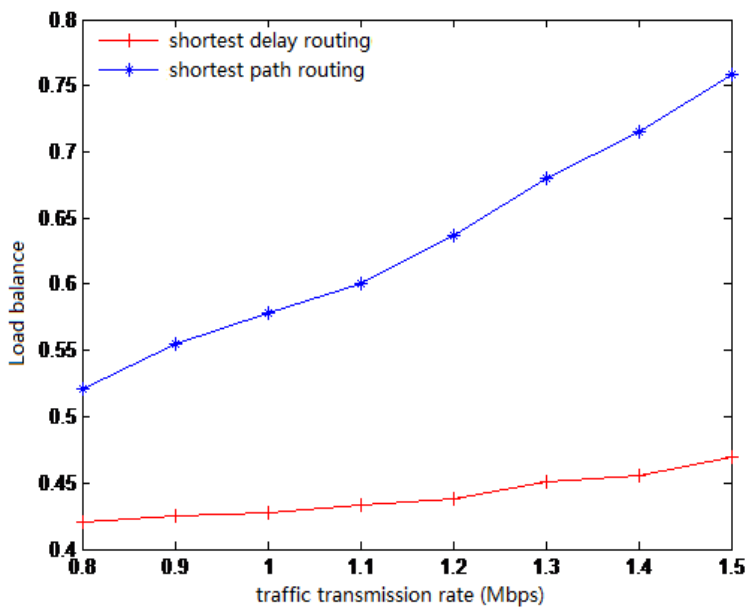

Fig. 6 The load balance

\section{Conclusions}

In this paper, a shortest delay routing for delay-sensitive traffic is proposed. In this routing, the queuing delay is calculated according to queuing theory and the retransmission delay caused by packet loss is considered when calculating the end-to-end delay for delay-sensitive traffic. By optimizing the delay cost, the end-to-end delay of delay-sensitive traffic can be reduced and its performance for throughput can be improved. It can be a meaningful routing method for the aerospace network with traffic diversity.

\section{Acknowledgements}

This work was supported in part by the Natural Science Foundation of Guangdong Province (2015A030310343) and Youth innovation project of Shenzhen Polytechnic(601722K19017 ).

\section{References}

[1] Werner M. A Dynamic Routing Concept for ATM-Based Satellite Personal Communication Networks[J]. IEEE Journal on Selected Areas in Communications, 1997, 15(8): 1636-1648.

[2] Hashimoto Y, Sarikaya B. Design of IP-based Routing in a LEO Satellite Network[C]. Proceedings of third international workshop on satellite-based information services (WOSBIS' 98), 1998: 81-88.

[3] Ekici E, Akyildiz I F, Bender M D. A Distributed Routing Algorithm for Datagram Traffic in LEO Satellite Networks[J]. IEEE/ACM Transactions on Networking, 2001, 9(2): 137-147.

[4] Kuang T, Ma R P. Darting: A Cost-effective Routing Alternative for Large Space-based Dynamic-topology Networks[C]. IEEE MILCOM 1995, 1995: 682- 686.

[5] Papapetrou E. Distributed on-demand Routing for LEO Satellite Systems [J]. Computer networks, 2007, 51(15): 4356-4376.

[6] Vatalaro F, Corazza G E, Caini C, et al. Analysis of LEO,MEO and GEO Global Mobile Satellite Systems in the Presence of Interference and Fading[J]. IEEE Journal on Selected Areas In Communications, 1995, 13(2): 291-300.

[7] Lee J W, Kim T W. Satellite over Satellite (SOS) Network: A Novel Concept of Hierarchical Architecture and Routing in Satellite Network[C]. 25th Annual IEEE Conference on Local Computer Networks, 2000: 392-399.

[8] Kim Y S, Park W J. Adaptive Routing in LEO Satellite Networks[C]. IEEE 2000 Vehicular Technology Conference. Tokyo: IEEE, 2000: 1983-1997.

[9] Nquyen H N, Jukan A. Approach to QoS-based Routing for Low Earth Orbit Satellite Networks[C]. Conference Record/IEEE Global Telecommunications Conference, 2000(2): 
1114-1118.

[10] Mohoricic M, Svigelj A, Kandus G. Traffic Class Dependent Routing in ISL Networks[J]. IEEE Transactions on Aerospace and Electronic Systems, 2004, 40(4): 1160-1172.

[11] Wenchao $\mathrm{Xu}$, Meng Jiang, Feilong Tang, Yanqin Yang. Network Coding-based Multi-path Routing algorithm in Two-layered Satellite Networks[J]. IET Communication, 2018, 12(1): 2-8.

[12] Yuan Yang, Mingwei Xu, Dan Wang, Yu Wang. Towards Energy-Efficient Routing in Satellite Networks[J]. IEEE Journal on Selected Areas in Communications, 2016, 34(12): 3869-3886.

[13] Xinmeng Liu, Zhuqing Jiang; Chonghua Liu, Shanbao He, Chao Li, Yuying Yang, Aidong Men. A low-complexity probabilistic routing algorithm for polar orbits satellite constellation networks[C]. 2015 IEEE/CIC International Conference on Communications in China (ICCC), 2015:1-5. 